\title{
Similarities, Differences, and Potential Synergies in the Mechanism of Action of Albumin Dialysis Using the MARS Albumin Dialysis Device and the CytoSorb Hemoperfusion Device in the Treatment of Liver Failure
}

\author{
Adrian Dominik Jan Stange \\ Department of Internal Medicine, Center for Extracorporeal Organ Support (CEOS), University of Rostock, Rostock, \\ Germany; Department of Internal Medicine, Section Nephrology, University Medicine Rostock, Rostock, Germany
}

\section{Keywords \\ MARS $^{\circledR} \cdot$ CytoSorb $^{\circledR} \cdot$ Albumin dialysis $\cdot$ Hemoperfusion . \\ Extracorporeal liver support}

\begin{abstract}
Introduction: Liver failure is characterized by compromised hepatic detoxification, protein synthesis, and metabolic derangements leading to an accumulation of a broad spectrum of water-soluble and lipophilic toxins as well as immune system mediators. Exploring complex detoxification mechanisms to therapeutically target those components, this article will focus on similarities, differences, and potential synergiesin themechanismofalbumin dialysisand hemoperfusion. Methods: An in vitro two-compartment model for the comparison of liver support techniques was used to compare MARS albumin dialysis modified with novel charcoal adsorbents to CytoSorb hemoperfusion with added hemodialysis for effects on marker molecule removal. Results: MARS and CytoSorb performed similar in the removal of water-soluble toxins. Ammonia removal was increased using CytoSorb. CytoSorb lead to a statistically significant reduction of albuminbound toxins, total bilirubin and subfractions. Bile acid removal was comparable. MARS demonstrated no removal of cytokines interleukin (IL)-6 and tumor necrosis factor-alpha
\end{abstract}

karger@karger.com www.karger.com/bpu

Karger ${ }^{\prime \prime} \div$

GOPEN ACCESS
(C) 2020 The Author(s)

Published by S. Karger AG, Basel

This is an Open Access article licensed under the Creative Commons Attribution-NonCommercial-4.0 International License (CC BY-NC) (http://www.karger.com/Services/OpenAccessLicense), applicable to the online version of the article only. Usage and distribution for commercial purposes requires written permission.
(TNF-a), whereas CytoSorb allowed for near complete removal. Notably, CytoSorb displayed $50 \%$ of lipophilic substance and cytokine removal during the first hour of treatment. Conclusion: Compared to MARS, CytoSorb hemoperfusion leads to an initially fast removal of cytokines, TNF-a and IL-6, as well as reduction of albumin-bound toxins such as indirect bilirubin and bile acids in our model. The initial removal is also associated with removal of albumin.

(c) 2020 The Author(s)

Published by S. Karger AG, Basel

\section{Introduction}

Liver Failure

Liver failure presents a clinical challenge with high short- and long-term mortality and is characterized by compromised hepatic detoxification and protein synthesis as well as metabolic derangements. When the liver seizes to detoxify endogenous and exogenous toxins and excrete bile into bile canaliculi, a broad spectrum of toxins accumulate primarily in the liver which then spill into

\section{Adrian Dominik}

Department of Internal Medicine, Center for Extracorporeal Organ Support (CEOS)

University of Rostock, Schillingallee 68

DE-18057 Rostock (Germany)

adrian.dominik.official@gmail.com

\section{Jan Stange}

Department of Internal Medicine, Section Nephrology

University Medicine Rostock, Schillingallee 68

DE-18057 Rostock (Germany)

jan.stange@web.de 
the systemic circulation causing autointoxication and affecting secondary organs $[1,2]$.

Inflammation and immunomodulation coordinate liver regeneration and are impaired in liver disease [2]. They play a major role in causing decompensation and secondary organ dysfunctions that define ACLF as the inflammatory pathophysiology progresses with hepatocellular damage and tissue remodeling induced by stellate cells toward fibrosis and cirrhosis $[3,4]$. The Chronic Liver Failure Consortium (CLIF) has revealed the significance of oxidized forms of albumin and pro-inflammatory cytokines, such as interleukin (IL)- 6 and tumor necrosis factor-alpha (TNF- $\alpha$ ) [5]. Cytokine effects include liver tissue regeneration and homeostasis [6]; however, they also sieve into the systemic circulation and lead to the development of hepatic encephalopathy [7] as well as problems associated with hyperdynamic circulation [8].

Albumin makes up for $50 \%$ of plasma proteins and has a significant role as a transport protein but also as a plasma expander, antioxidant, and immunomodulating compound [9]. Transport functions are realized via its tertiary structure providing several binding sites for endogenous or exogenous compounds ranging from bilirubin, bile acids, or hormones to metals, anions, fatty acids, and drugs [10]. Transport functions can be assessed, for example, by analyzing albumin-binding capacity $(\mathrm{ABiC}$; remaining binding capacity at Sudlow II binding site) which links the MELD Score used for liver failure severity assessment to a single biomarker. This highlights the importance of albumin-bound toxins for short-term prognosis [11] and provides an increasing rationale to intervene with therapies capable of removing albumin-bound toxins.

\section{Extracorporeal Organ Support}

In order to address the complexity of toxic agents and immune system mediators in the systemic circulation in ACLF, the medical community is currently exploring complex detoxification mechanisms to therapeutically target those components. Conventional hemodialysis is limited in the removal of protein-bound toxins and cytokines, which in turn led to the development of hemoperfusion devices and albumin dialysis techniques [12].

In albumin dialysis, patients' blood is being dialyzed against albumin-enriched dialysate. This allows the transfer of albumin-bound toxins from the patient to dialysate which is discarded in single-pass albumin dialysis or can be regenerated by different adsorbents in recirculating systems [13]. MARS (molecular adsorbent recirculating system) has been introduced into clinic as an albumin dialysis technique. The biggest clinical trial on MARS "RELIEF," however, showed no significant improvements in survival compared to standard medical therapy. Discussed drawbacks of this study were treatment frequency, detoxification capacity of adsorbents, as well as cytokine removal options $[2,14-16]$. As a means of hemoadsorption, the CytoSorb adsorbent is a hemoperfusion device marketed for the extracorporeal removal of cytokines and proteins from blood in a concentration-dependent manner [17]. Recently, a reduction of liver failure-related toxins has been added to the intended use of CytoSorb in the EU [18].

\section{Aim}

The aim of the present article was to compare albumin dialysis using MARS to the CytoSorb hemoperfusion device in an established in vitro two-compartment model for the comparison of liver support techniques. Further, this article will focus on similarities, differences, and potential synergies in the mechanism of these therapies and could outline a pathway to a clinical trial addressing acute liver failure or acute decompensation of chronic liver disease combined with severe inflammation.

\section{Material and Methods}

\section{Two-Compartment Model}

An established in vitro 2-compartment model for the comparison of different albumin dialysis techniques was developed in our department, which mimics pathologic toxin levels in patients during liver failure [19]. Briefly, compartment 1 is made up from $1 \mathrm{~L}$ of human plasma spiked with liver failure toxins including watersoluble (ammonia, $\mathrm{Cr}$, and urea) and albumin-bound toxins (conjugated [direct] bilirubin, unconjugated [indirect] bilirubin, bromosulphthalein, primary bile acid, and chenodesoxycholic acid). Dynamic processes of toxin accumulation and redistribution from the extra-vascular space are accounted for in this model using continuous infusion of compartment 2 solution made up from aforementioned toxins. Currently, the used 2-compartment model does not account for cytokine removal and redistribution; however it was modified by the addition of $150 \mu \mathrm{g} / \mathrm{L}$ recombinant human TNF- $\alpha$ (BioLegend, San Diego, CA, USA) and $150 \mu \mathrm{g} / \mathrm{L}$ recombinant human IL-6 (BioLegend) to compartment 1 to give first insights. Both compartment solutions are prepared 1 day before the experiment and kept cool at $4^{\circ} \mathrm{C}$ overnight. This prevents clotting of plasma proteins and disintegration of thermo- and photosensitive substances. Further 5000 units of Heparin (Ratiopharm, Ulm, Germany) were added to the compartment 1. 
Fig. 1. Experimental setup for MARS and CytoSorb treatments with added hemodialysis circuit. Displayed are denoted filters (MARS Flux, VitaPES, and CytoSorb hemoperfusion device), compartment solutions (compartment 1, compartment 2), adsorbent (Hepalbin), flow rates $(150 \mathrm{~mL} /$ min plasma flow, $50 \mathrm{~mL} / \mathrm{min}$ recirculation flow, diaylsis flow $2,000 \mathrm{~mL} / \mathrm{h}$, and ultrafiltration $90 \mathrm{~mL} / \mathrm{h}$ ), and sample point $\mathrm{Pi}$. $\mathrm{Pi}$, patient inlet.

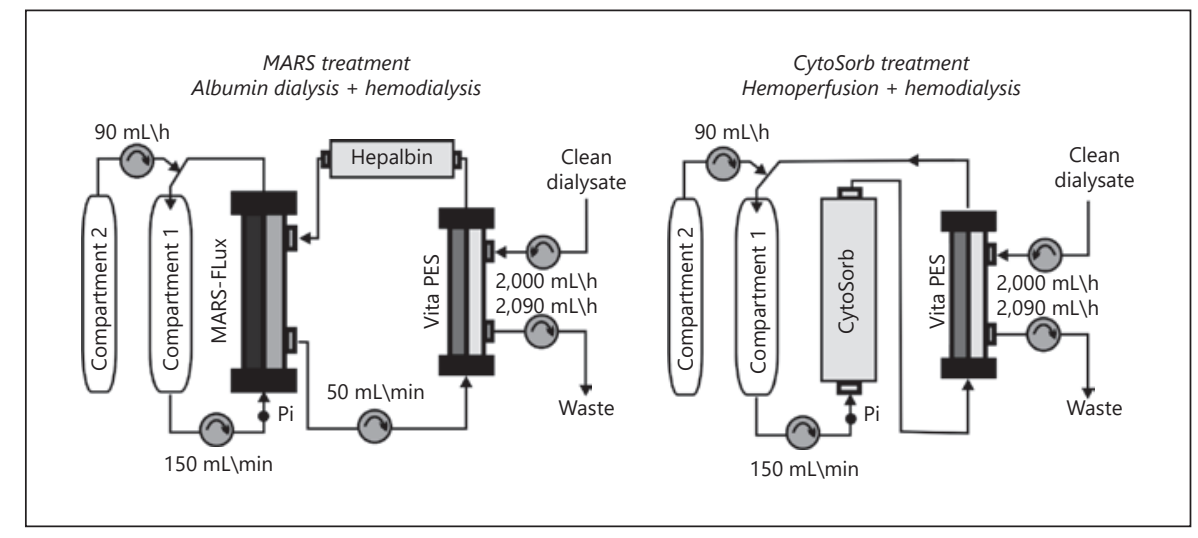

\section{In vitro Treatment Setup}

Albumin dialysis using MARS and hemoperfusion using CytoSorb is performed using a hemodialysis platform setup according to the manufacturer's manual (NxStage System One, Lawrence, MA, USA). For Albumin dialysis, a MARS Flux (Gambro, Rostock-Warnemünde, Germany) membrane is used as primary hemofilter. A low-flux membrane VitaPES (VitaPES 130 LF; MTP Medical Technologies $\mathrm{GmbH}$, Pirna, Germany) is used in both setups for hemodialysis.

Dialysis solution is MultiBic (2 $\mathrm{mm}$ potassium; Fresenius, Bad Homburg, Germany). Infusion of compartment 2 solution is realized by an infusion pump (Volumed VP5000; Fresenius, Bad Homburg, Germany). Flow rates are $150 \mathrm{~mL} / \mathrm{min}$ plasma flow, $50 \mathrm{~mL} /$ min recirculation flow (only in albumin dialysis), $90 \mathrm{~mL} / \mathrm{h}$ continuous infusion of compartment 2 solution, 2,000 mL/h dialysate flow, and $90 \mathrm{~mL} / \mathrm{h}$ ultrafiltration (started at $t=120 \mathrm{~min}$ ). Samples are collected from patient inlet $(\mathrm{Pi})$, compartment $1(\mathrm{~K} 1)$, and compartment 2 (K2). Time points for sample taking are $0,10,30,60$, $120,180,240,300$, and $360 \mathrm{~min}$. Sample volumes are $3 \mathrm{~mL}$ at each sample and time point $(3 \times 1 \mathrm{~mL}$ aliquots); $5 \mathrm{~mL}$ were taken extra at time points K1, 30, 180, and $360 \mathrm{~min}$. Samples were directly frozen at $-30^{\circ} \mathrm{C}$. As part of the rinsing solution remains inside the system, initial concentrations at sample point $\mathrm{Pi}(0 \mathrm{~min})$ differ from compartment 1 concentrations (K1) due to dilution effects.

\section{Albumin Dialysis Using MARS}

The hemofiltration and dialysis circuit were operated by the dialysis platform and the recirculation circuit was operated by a MARS Monitor (Gambro, Rostock-Warnemünde, Germany) and are prepared according to the manufacturer's manual. The recirculation circuit contains albumin-enriched dialysate $(400 \mathrm{~mL} 20 \%$ human serum albumin [HSA] in dialysate solution). Due to known saturation effects of MARS adsorbents diaMARS AC250 and diaMARS IE 250 during MARS treatments [20-23], MARS adsorbents were exchanged with a Hepalbin adsorbent (Hepalbin Cluster ${ }^{12}$; Albutec GmbH, Rostock, Germany), previously shown to have improved adsorbent capacity [24]. This allows for a comparison of albumin dialysis and hemoperfusion concerning toxin transfer.

First, all connections of hemofiltration and dialysis circuits are clamped. Then the recirculation circuit is rinsed using $0.9 \% \mathrm{NaCl}$ according to the manufacturer's manual (MARS monitor). Now, $400 \mathrm{~mL}$ of $20 \%$ HSA (stabilizer depleted) is added to the recirculation circuit. The next step includes the clamping of the recircula- tion connections and rinsing of the hemofiltration and dialysis circuit according to the on-screen instructions of the used dialysis platform NxStage System One. Finally, all clamps are detached and compartments 1 and 2 are connected.

\section{Hemoadsorption Using CytoSorb}

As MARS includes a hemodialysis circuit for the removal of water-soluble toxins and CytoSorb works as a perfusion device, a hemodialysis circuit is added to the CytoSorb experiments in order to exclude effects of marker molecule accumulation. The dialysis circuit was operated in conventional hemodialysis mode using the dialysis platform NxStage System One and was set up according to the manufacturer's manual, which includes rinsing of the system using $0.9 \% \mathrm{NaCl}$ following the on-screen instructions. Finally, compartments 1 and 2 are connected (Fig. 1).

\section{Substances}

Twenty percent HSA which contains the stabilizers N-acetyltryptophanate $(10-16 \mathrm{mmol} / \mathrm{L})$ and octanoate $(10-16 \mathrm{mmol} / \mathrm{L})$ was purchased from CSL Behring (King of Prussia, PA, USA). Stabilizer-depleted albumin was provided by Albutec (Albutec $\mathrm{GmbH}$, Rostock, Germany). Chenodesoxycholic(bile-) acid, Cr, conjugated and unconjugated bilirubin, bromosulpthalein, ammonium chloride, and urea were purchased from Sigma Aldrich (St. Louis, MO, USA). Dialysate solution was MultiBic ${ }^{\circledR}(2 \mathrm{mmol} / \mathrm{L}$ potassium), purchased from Fresenius Medical Care (Bad Homburg, Germany). Sodium hydroxide and sodium chloride solutions were purchased from Roth (Karlsruhe, Germany).

\section{Biochemical Analysis}

Substance concentrations were analyzed by photometric assay using Cobas Mira Plus (Roche, Basel, Switzerland). Ammonia was measured by an enzymatic reaction of alpha-ketoglutarate and NADPH using glutamate dehydrogenase to give L-glutamate + $\mathrm{NADP}^{+}$. Urea was measured by an enzymatic UV method where urea is converted to $\mathrm{NH}_{4}{ }^{+}$and $\mathrm{HCO}_{3}{ }^{-} ; \mathrm{HCO}_{3}{ }^{-}$reacts with alphaketoglutarate and $\mathrm{NADH}$ using glutamate dehydrogenase to give L-glutamate and $\mathrm{NAD}^{+}$. Cr was measured using the Jaffé method. Albumin was measured according to Doumas et al. [25]. Concentrations of bile acids were analyzed by an enzymatic method as described by Mashige et al. [26, 27]. A modified method of Jendrassik and Grof was used for analysis of total and conjugated bilirubin [28]. The subtraction of conjugated bilirubin from total bil- 


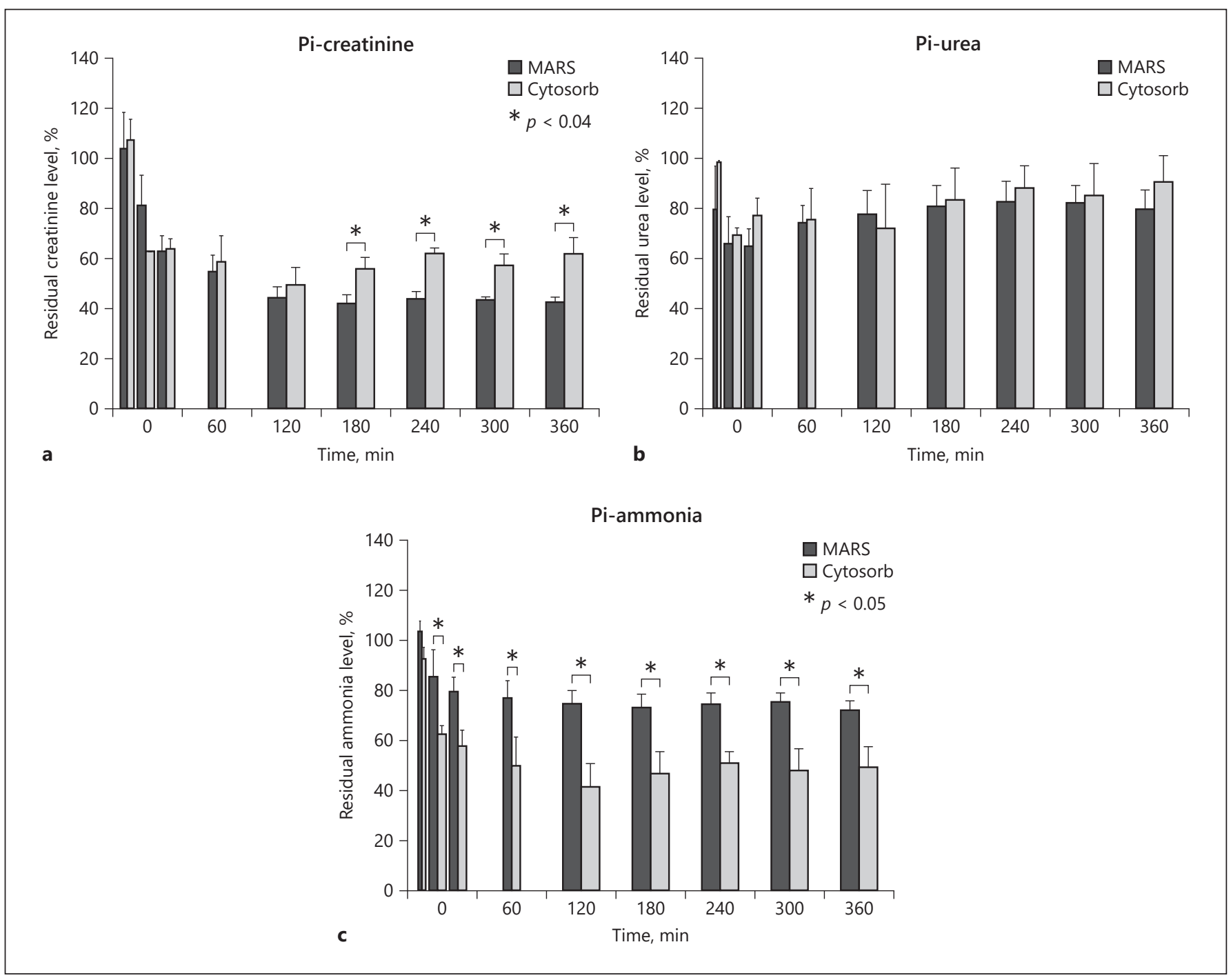

Fig. 2. Residual levels of water-soluble marker molecules $\mathrm{Cr}(\mathbf{a})$, urea (b), and ammonia (c) referenced against initial compartment 1 concentrations over time $(n=3)$. Pi, patient inlet.

irubin concentration results in the concentration of unconjugated bilirubin. Concentrations of IL- 6 and TNF- $\alpha$ were measured by ELISA using the respective kits (BioLegend ELISA MaxTM, San Diego, CA, USA). The ABiC test was performed as described by Klammt et al. [11, 29].

Data Analysis

All statistical analyses were performed using Excel (Version 2007; Microsoft Office, Microsoft, Redmond, WA, USA) and SigmaPlot (Version 11.0; Systat Software, Chicago, IL, USA). Results are referenced against compartment 1 concentrations for $100 \%$ reference and displayed as bar charts with mean and standard deviation for comparison of residual amounts. Statistical analysis was performed using an independent 2-tailed $t$ test.

\section{Results}

Measured concentrations for water-soluble toxins and albumin-bound toxins are referenced against compartment 1 concentrations as a $100 \%$ reference. This allows for the comparison of residual levels. $\mathrm{ABiC}$ values are being reported without further calculations.

\section{Water-Soluble Toxins}

Looking at remaining toxin concentrations, MARS allowed for significant higher removal of Cr during the last $3 \mathrm{~h}$ of treatment. Urea removal displayed no difference; however, ammonia removal was significantly 


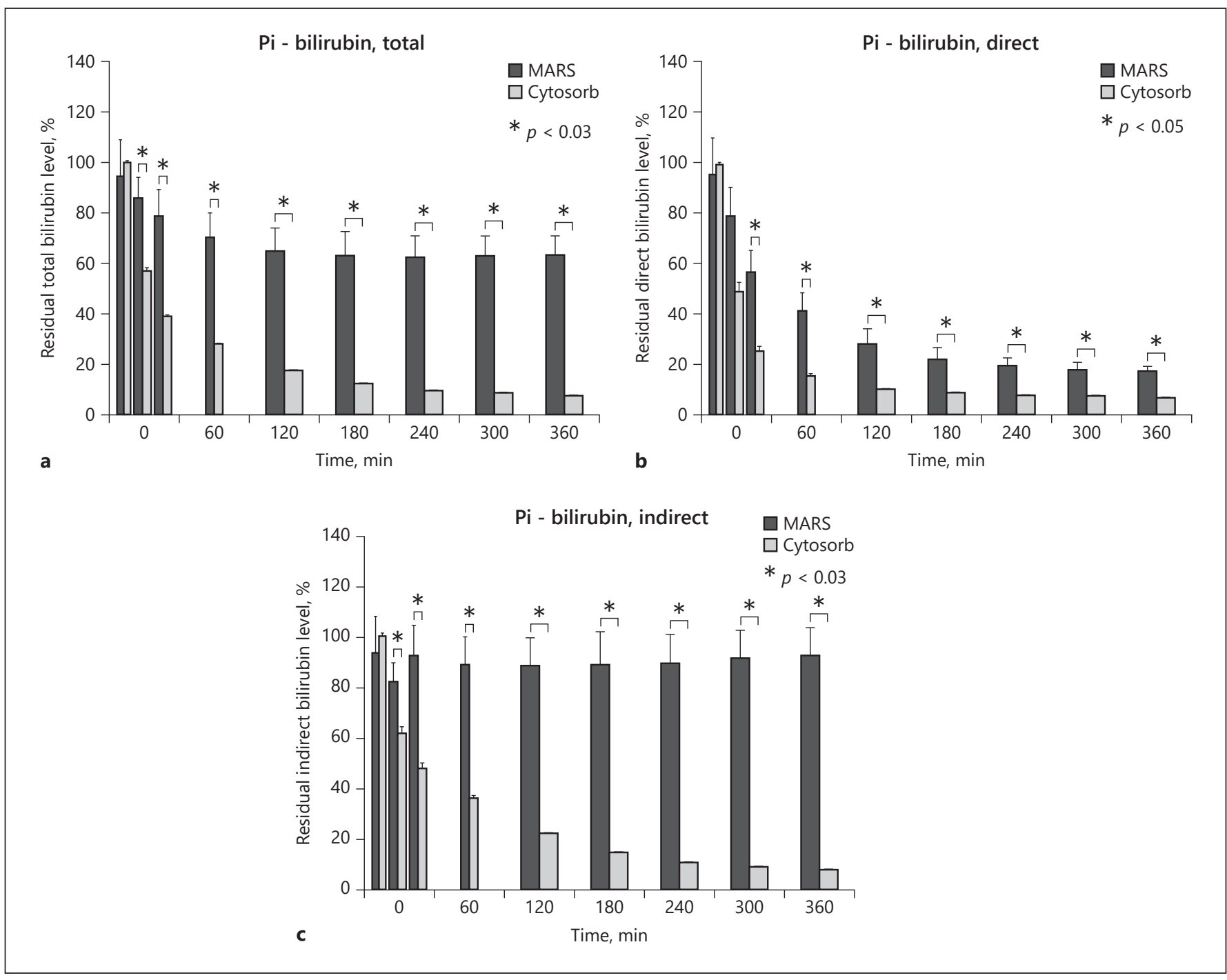

Fig. 3. Residual levels of albumin-bound marker molecule bilirubin referenced against initial compartment 1 concentrations for total bilirubin (a) as well as subfractions direct bilirubin (b) and indirect bilirubin (c) over time $(n=3)$. Pi, patient inlet.

increased over the 6-h treatment using CytoSorb (Fig. 2).

\section{Albumin-Bound Toxins}

Using the CytoSorb hemoperfusion device lead to a statistically significant reduction of total bilirubin and subfractions compared to albumin dialysis using MARS. Whereas direct bilirubin concentrations could be reduced by both systems efficiently after $6 \mathrm{~h}$ (MARS 16\%; CytoSorb 7\%), only CytoSorb was able to reduce indirect bilirubin levels (MARS 92\%, CytoSorb 8\%) (Fig. 3). The CytoSorb device allows for a faster initial removal of chenodeoxycholic acid compared to albumin dialysis using MARS; however, both techniques displayed a reduction down to approximately $10-15 \%$ of initial concentrations after $6 \mathrm{~h}$ of experiment (Fig. 4).

\section{Cytokines (IL-6 and TNF- $\alpha$ )}

MARS demonstrated no removal of cytokines IL-6 and TNF- $\alpha$, whereas CytoSorb allowed for a near complete removal after $6 \mathrm{~h}$ of treatment (4\% remaining IL-6; $8 \%$ remaining TNF- $\alpha$ ). Notably, treatment using the CytoSorb device shows the main reduction appearing within the first half an hour of treatment (Fig. 5). 


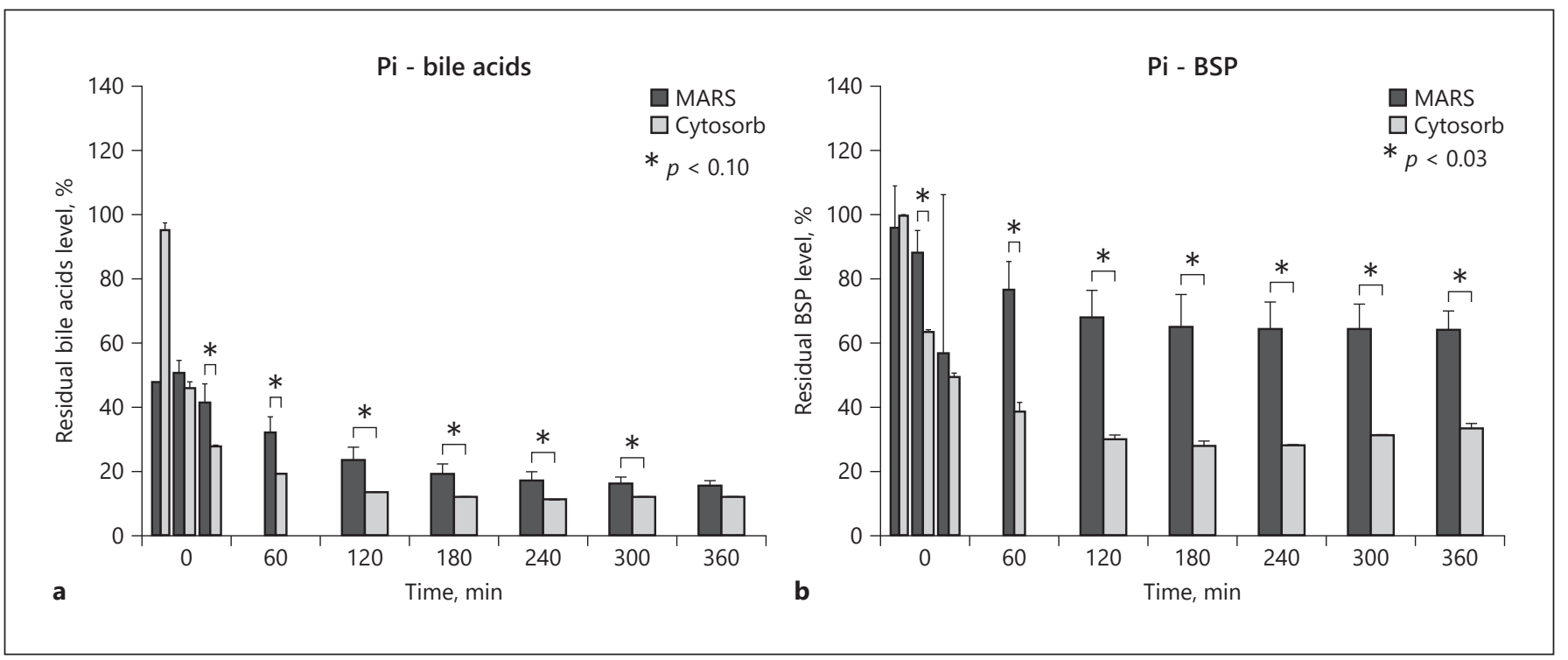

Fig. 4. Residual levels of albumin-bound marker molecules bile acids (chenodeoxycholic acid; a) and bromosulphthalein (BSP; b) referenced against initial compartment 1 concentrations over time $(n=3)$. Pi, patient inlet.

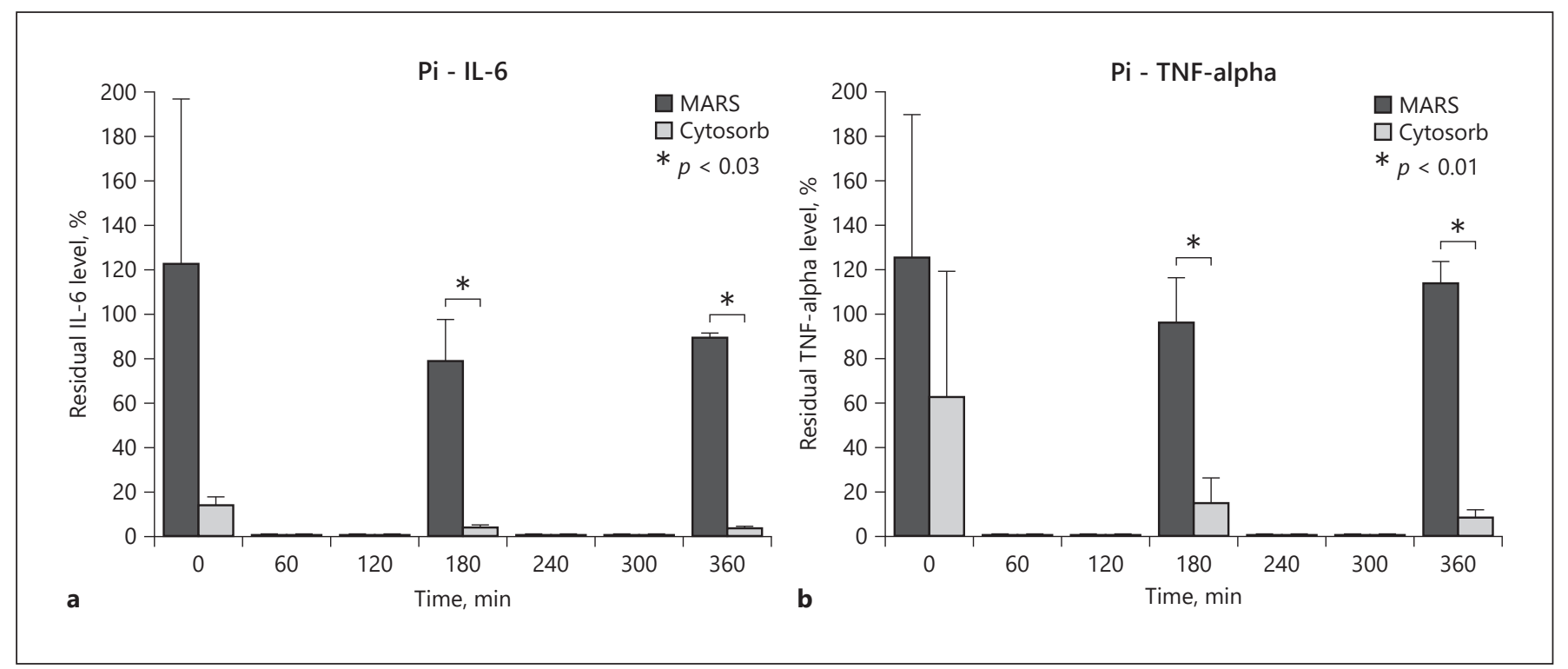

Fig. 5. Residual levels of IL-6 (a) and TNF- $\alpha$ (b) referenced against initial compartment 1 concentrations over time $(n=3)$. IL, interleukin; TNF- $\alpha$, tumor necrosis factor-alpha; Pi, patient inlet.

\section{Albumin $+A B i C$}

Albumin dialysis using MARS lead to a significant increase of albumin $(+45 \pm 13 \%$ in reference to compartment 1 concentrations) as a consequence of continuous infusion of the toxin cocktail. This increase was not observed in the CytoSorb tests $(-30 \pm 6 \%$ in reference to compartment 1 concentrations) over $6 \mathrm{~h}$ of experiment time. Albumin-binding capacity was increased by $25 \%$ $(p<0.01)$ using MARS $\left(t_{0}=45 \pm 2 \% ; t_{360}=71 \pm 3 \%\right.$; $p<$ $0.0003)$ compared to nonsignificant trend using CytoSorb $\left(t_{0}=46 \pm 2 \% ; t_{360}=49 \pm 2 \% ; p<0.14\right)$. 


\section{Discussion: Effects on Marker Molecule Removal and Clinical Implications}

\section{Water-Soluble Toxins}

Hemodialysis techniques have been established for the removal of small water-soluble toxins from blood. Using a low-flux membrane, water-soluble toxins move along their concentration gradient from the patient circuit into a clean dialysate solution and are subsequently discarded into a waste bag. CytoSorb is frequently used in patients with severe inflammation in combination with dialysis, which is why we decided to apply CytoSorb in this test in combination with dialysis using identical flow rates in order to be able to differentiate differences also for watersoluble substances. Slow dialysate flow was used as these therapies are usually applied continuously and to eliminate the effects of different concentration gradients between MARS and the CytoSorb/dialysis setup: In MARS, hemodialysis is realized by a secondary dialyzer within the albumin recirculation circuit. At slow dialysis flow rates, the concentration of small water-soluble molecules by the dialysis outlet is equivalent to the plasma concentration.

Looking at the removal of urea, we did not observe any significant differences. The significantly higher removal of ammonia was surprising given its small molecular size and high water solubility with no binding to albumin. In MARS, experiments ammonia is cleared from the patient circuit via passage into the albumin dialysis circuit (MARS Flux membrane as primary toxin transfer barrier) and subsequent hemodialysis of this circuit (VitaPES membrane as secondary toxin transfer barrier), whereas hemodialysis in CytoSorb experiment benefits from having no secondary circuit, which could explain better removal of ammonia. However, this observation needs further analysis as it is not supported by the removal of other small water-soluble toxins, but confirmed earlier observations published by Büttner et al. [30] arguing that CytoSorb might eliminate other nitrogenic substances which lead to indirect ammonia reduction in plasma. This may be enhanced by plasma enzymes that can actively convert ammonia into other molecules (such as reverse glutamate dehydrogenase or glutamine synthase) and back. It is possible that the activity of the glutamate dehydrogenase or of the glutamine synthase is increased in the vicinity of a hydrophobic polymer. The removal of $\mathrm{Cr}$ was better using MARS which was less surprising, since charcoal as employed in the modified MARS setup is an excellent adsorbent for $\mathrm{Cr}$ [31].

In vitro: MARS versus CytoSorb in Liver Failure

\section{Clinical Implications}

As the removal of water-soluble toxins is an established therapeutic intervention by conventional hemodialysis and CytoSorb can be run in series with a low-flux dialyzer on a CVVH machine. There are multiple benefits of integrating dialysis in the extracorporeal treatment of patients with ACLF. First of all, in a vast majority of patients with ACLF, renal insufficiency is the most frequent complication. Second, the removal of nitrogen substances can assist in reducing ammonia in vivo. Ammonia is one of the water-soluble toxins in liver failure, being in part responsible for the development of hepatic encephalopathy.

\section{Albumin-Bound Toxins}

During liver diseases, cholic acid, chenodeoxycholic acid, and deoxycholic as well as their conjugates represent the vast majority of bile acids in circulation $(>95 \%)$ and can accumulate to pathological concentrations. A recent study on bile acid removal by extracorporeal therapies revealed HSA as the main transporter for deoxycholic-, chenodeoxycholic-, and lithocholic acid and further showed that bile acid removal by means of blood purification is strongly influenced by their hydrophobicity and, therefore, binding to albumin [32]. Interestingly, different profiles and patterns of serum bile acids have been shown to discriminate between health and disease and can hint at underlying pathology in liver disease [33]. Bile acid homeostasis is closely regulated not to exceed physiological concentrations by a complex interplay of synthesis, metabolism, and excretion. Bile acids can interact with the nuclear farnesoid X receptor (FXR) and cell-surface G-protein-coupled receptor TGR5 which are involved in maintaining metabolic homeostasis concerning energy, lipid, and glucose metabolism [34]. Main ligands of FXR are chenodeoxycholic acid as well as cholic acid. Further, bile acids have been shown to induce inflammatory-associated genes in hepatocytes and induce stellate cell proliferation contributing to inflammation and hepatotoxic effects of elevated bile acids [35], which can be further aggravated by pro-inflammatory cytokine signaling [34].

In our experiments, the CytoSorb hemoperfusion device leads to a markedly initial increase of the reduction of lipophilic toxins such as direct and indirect bilirubin, chenodeoxycholic acid, as well as the liver function marker bromosulphthalein when compared to MARS, which is obviously an effect of direct hemoperfusion. Only after $6 \mathrm{~h}$, the concentrations in the model tend to be comparable for direct bilirubin, bromosulphthalein, and bile ac-

Blood Purif 2021;50:119-128 
ids, all molecules with a binding constant of around $10^{-4}$ to $10^{-5}$ [36]. MARS showed the capacity to remove direct bilirubin; however, it did not allow for a reduction of indirect bilirubin, as observed by others $[2,16]$. Remarkably, unconjugated bilirubin decreased significantly during CytoSorb treatment. From our data, it can be assumed that CytoSorb, at least initially, adsorbs highly albuminbound substances such as unconjugated bilirubin (binding constant $10^{-7}$ and higher) or even irreversibly bound toxins such as delta bilirubin at a measurable amount.

We observed a loss of albumin in the hemoperfusion group within the first $60 \mathrm{~min}$, followed by an increase, indicating saturation. Interestingly, the binding capacity of albumin did not increase over the first $6 \mathrm{~h}$. Notably, the 2-compartment model only contains human plasma and thereby differs from in vivo conditions due to the absence of whole blood. Whether the observed reduction of albumin is specific for more oxidized forms of albumin should be investigated in further studies. Clinically, the loss of albumin is not higher than in other newer apheresis technologies investigated for detoxification in ACLF such as DIALIVE, which works as an albumin apheresis technique currently in clinical trial [37].

\section{Clinical Implications}

The inability of the MARS membrane to facilitate removal of indirect bilirubin has been investigated in a previous study and can be overcome by introducing novel membranes [38]. Bile acid reduction after $360 \mathrm{~min}$ was comparable for both systems. This indicates no technological superiority in lipophilic toxin removal of CytoSorb hemoperfusion to MARS albumin dialysis if novel membranes are employed for MARS.

Insights into cholestatic liver injury revealed high levels of bile acids to induce the production of pro-inflammatory mediators in the liver which lead to neutrophil chemotaxis and associated killing of injured hepatocytes [39]. It can be assumed that the change of the pattern of bile acids from more lipophilic toxic compounds toward more hydrophilic bile acids is an important mechanism of action of effective detoxification devices in liver failure as this could counteract pathophysiologic processes. Recently, obeticholic acid (OCA) as a derivate of chenodeoxycholic acid was synthesized in search for a potent FXR activator in the treatment of NASH or primary biliary cirrhosis. OCA displays a 100 -fold higher affinity to the FXR receptor and is associated with anti-fibrotic, anti-inflammatory, and tissue regeneration in preclinical studies [40]. OCA reduces conversion of cholesterol to bile acids and further downregulates bile acid transport- ers responsible for hepatic uptake [41]. The FLINT trial (clinical trial on patients with non-cirrhotic, nonalcoholic steatohepatitis to assess treatment with OCA given orally), however, revealed pruritus as well as an increase in serum cholesterol as complications of treatment [42]. Here, we speculate that removal of primary bile acids like chenodeoxycholate by means of extracorporeal blood purification could further ameliorate treatment of liver diseases where lipophilic toxins mediate disease severity or are accumulating due to pharmaceutical intervention as seen in the FLINT trial [42]. A potential synergy of extracorporeal removal of lipophilic bile acids with administration of FXR agonist therapy appears worth further clinical investigation. Albumin dialysis has already been shown to reduce pruritogens and signs of pruritus in patients[43]. Last but not least, dosing regimens of administered drugs need to be analyzed during extracorporeal blood purification treatments in order to guarantee effective treatment as adsorption is indicated for lipophilic or protein-bound drugs. However, this is a challenge which is similar in other therapies employing albumin apheresis (plasma exchange, DIALIVE, etc.), and experienced critical care specialists have developed algorithms to compensate for removal of medications by extracorporeal therapies [43].

\section{Cytokines}

Rozga et al. [41] classified cytokines and immunecompetent mediators according to their size and biological activity in liver failure, whereas smaller sized pro-inflammatory cytokines like IL-6 (22-27 kDa), TNF- $\alpha$ (trimer $51 \mathrm{kDa})$, transforming growth factor-beta $1(44 \mathrm{kDa})$, and endotoxin components (lipid A, $10-20 \mathrm{kDa}$ [42]) or anaphylatoxins (C3a, $10 \mathrm{kDa}$ ) contribute to disease severity by being negative regulators of hepatocyte proliferation, larger molecular sized hepatocyte growth factor (190 kDa), immunoglobulins (IgG, $150 \mathrm{kDa}$; IgA, 320 $\mathrm{kDa}$; and IgM, $900 \mathrm{kDa}$ ), and components of the complement system $(30-200 \mathrm{kDa})$ contribute to physiological function and hepatocyte recovery [44]. This is aggravated by decreased elimination and increased synthesis of cytokines observed in chronic liver failure [45]. With this work, Rozga laid the groundwork for more recent versions of albumin apheresis devices, such as DIALIVE which target a spectrum of molecules similar to CytoSorb [37].

Looking at cytokine removal, only little IL- 6 was removed and MARS was unable to clear TNF- $\alpha$ from the circulation. In future studies, rebound and redistribution phenomena should be accounted for in the two-compart- 
ment model. Effects on additional negative and positive regulators of hepatocyte proliferation should also be examined in further studies.

\section{Clinical Implications}

In a previous study [38], we were able to show that increasing the pore size of membranes in albumin dialysis can increase removal of albumin-bound toxins like indirect bilirubin and introduces the option of near complete cytokine removal for IL- 6 and TNF- $\alpha$ in vitro when compared to the MARS Flux membrane. Notably, the simple addition of albumin to dialysate solution increases clearance of cytokines in vitro, indicating potential of albumin dialysis for immunomodulatory interventions [46]. However, data confirmed that standard high-flux dialysis membranes such as the MARS Flux cannot compete with the cytokine removal observed with CytoSorb.

\section{Conclusion}

Although CytoSorb is intended to be used as a whole blood hemoperfusion device, it is believed that the twocompartment plasma model provided good insights into the removal rates with respect to comparing MARS versus CytoSorb, since possible deviations caused by the use of plasma should apply for both systems. Compared to MARS, CytoSorb hemoperfusion leads to an initially fast removal of cytokines TNF- $\alpha$ and IL- 6 as well as reduction of albumin-bound toxins such as indirect bilirubin and bile acids in our model. The initial removal is also associated with the removal of some albumin. Clinically, the loss can be compensated by replacement with fresh albu$\min$.

\section{Limitations of This Study}

The present study is based on applying an in vitro two-compartment human plasma-based model developed for the comparison of liver support techniques to two different means of treatment: albumin dialysis and hemoadsorption. Whereas the albumin dialysis circuit is in indirect plasma contact due to a hemofilter membrane separation, hemoadsorption is in direct plasma contact.

Only human plasma is used within this model, and effects in whole blood might differ. Cytokines added to the compartment 1 solution are not accounted for concerning dynamic redistribution phenomena.

\section{Acknowledgement}

The technical assistance of Helga Weiss-Reining is gratefully acknowledged.

\section{Statement of Ethics}

As this was an in vitro study, no ethical approval was required.

\section{Disclosure Statement}

Jan Stange is the chairman of the board at Albutec $\mathrm{GmbH}$ and a consultant to CytoSorbents Deutschland GmbH. CytoSorbents $\mathrm{GmbH}$ was not involved in planning, conducting, and data analysis of the performed tests or writing of the manuscript. Jan Stange consulted CytoSorbents $\mathrm{GmbH}$ for discussion purposes. The company was allowed to read before submission but had no rights to deny it since the study was only supported in part. All other authors have declared no competing interests.

\section{Funding Sources} $\mathrm{GmbH}$.

This work was supported by a grant provided by CytoSorbents

\section{Author Contributions}

Adrian Dominik performed the in vitro testing, data analysis, and graphical representation of data. Adrian Dominik and Jan Stange contributed equally in writing of the manuscript.

\section{References}

1 Grek A, Arasi L. Acute liver failure. AACN Adv Crit Care. 2016 Oct;27(4):420-9.

2 Ilonen I, Koivusalo A-M, Repo H, Höckerstedt $\mathrm{K}$, Isoniemi $\mathrm{H}$. Cytokine profiles in acute liver failure treated with albumin dialysis. Artif Organs. 2007 Aug;0(0):070802063815003???.
3 Hardy T, Oakley F, Anstee QM, Day CP. Nonalcoholic fatty liver disease: pathogenesis and disease spectrum. Annu Rev Pathol Mech Dis. 2016 May;11(1):451-96

4 Caligiuri A, Gentilini A, Marra F. Molecular pathogenesis of NASH. Int J Mol Sci. 2016 Sep;17(9):1575.
5 Clària J, Stauber RE, Coenraad MJ, Moreau R, Jalan R, Pavesi M, et al. Systemic inflammation in decompensated cirrhosis: characterization and role in acute-on-chronic liver failure. Hepatology. 2016;64(4):1249-64. 
6 Cressman DE, Greenbaum LE, DeAngelis RA, Ciliberto G, Furth EE, Poli V, et al. Liver failure and defective hepatocyte regeneration in interleukin-6-deficient mice. Science. 1996 Nov;274(5291):1379-83.

7 Montoliu C, Piedrafita B, Serra MA, del Olmo JA, Urios A, Rodrigo JM, et al. IL-6 and IL-18 in blood may discriminate cirrhotic patients with and without minimal hepatic encephalopathy. J Clin Gastroenterol. 2009 Mar; 43(3):272-9.

8 Liu H, Lee SS. Acute-on-chronic liver failure: the heart and systemic hemodynamics. Curr Opin Crit Care. 2011 Apr;17(2):190-4.

9 Garcia-Martinez R, Caraceni P, Bernardi M, Gines P, Arroyo V, Jalan R. Albumin: pathophysiologic basis of its role in the treatment of cirrhosis and its complications. Hepatology. 2013 Nov;58(5):1836-46.

10 Ferrer R, Mateu X, Maseda E, Yébenes JC, Aldecoa C, De Haro C, et al. Non-oncotic properties of albumin. A multidisciplinary vision about the implications for critically ill patients. Expert Rev Clin Pharmacol. 2018 Feb; 11(2):125-37.

11 Klammt S, Mitzner S, Stange J, Brinkmann B, Drewelow B, Emmrich J, et al. Albumin-binding function is reduced in patients with decompensated cirrhosis and correlates inversely with severity of liver disease assessed by model for end-stage liver disease. Eur J Gastroenterol Hepatol. 2007 Mar;19(3):257-63.

12 Sen S, Williams R. New liver support devices in acute liver failure: a critical evaluation. Semin Liver Dis. 2003;23(3):283-94.

13 Nevens F, Laleman W. Artificial liver support devices as treatment option for liver failure. Best Pract Res Clin. Gastroenterol. 2012 Feb; 26(1):17-26.

14 Bañares R, Nevens F, Larsen FS, Jalan R, Albillos A, Dollinger M, et al. Extracorporeal albumin dialysis with the molecular adsorbent recirculating system in acute-on-chronic liver failure: the RELIEF trial. Hepatology. 2013 Mar;57(3):1153-62.

15 Sen S, Davies NA, Mookerjee RP, Cheshire LM, Hodges SJ, Williams R, et al. Pathophysiological effects of albumin dialysis in acuteon-chronic liver failure: a randomized controlled study. Liver Transpl. 2004 Sep;10(9): 1109-19.

16 Stadlbauer V, Krisper P, Aigner R, Haditsch B, Jung A, Lackner C, et al. Effect of extracorporeal liver support by MARS and prometheus on serum cytokines in acute-onchronic liver failure. Crit Care. 2006;10(6): R169.

17 The Adsorber, CytoSorb. [Online]. [cited 2019 Apr 24]. Available from: https://cytosorb-therapy.com/the-adsorber/.

18 Fields of application, CytoSorb. [Online] [cited 2019 May 27]. Available from: https:// cytosorb-therapy.com/the-therapy/fields-ofapplication/.
19 Stiffel M, Dammeier N, Schmidt S, Szyszkowitz T, Sikole M, Sieveking C, et al. 24-hour performance of albumin dialysis assessed by a new two-compartment in vitro model. Ther Apher Dial. 2014 Feb;18(1):79-86.

20 Evenepoel P, Laleman W, Wilmer A, Claes K, Kuypers D, Bammens B, et al. Prometheus versus molecular adsorbents recirculating system: comparison of efficiency in two different liver detoxification devices. Artif Organs. $2006 \mathrm{Apr} ; 30(4): 276-84$.

21 Gerth HU, Pohlen M, Thölking G, Pavenstädt $\mathrm{H}$, Brand $\mathrm{M}$, Hüsing-Kabar A, et al. Molecular adsorbent recirculating system can reduce short-term mortality among patients with acute-on-chronic liver failure-A retrospective analysis. Crit Care Med. 2017 Oct;45(10): 1616-24.

22 Tan HK. Molecular adsorbent recirculating system (MARS). Ann Acad Med. 2004 May; 33(3):329-35

23 Gong D, Cruz D, Ronco C. Depurative capacity of molecular adsorbent recycling system (MARS): a focus on bilirubin removal. Int J Artif Organs. 2008 Dec;31(12):875-81.

24 Dominik A, Stange J, Baumann AK, Pfensig C, Suraj M, Ibrahim B, et al. Targeting albumin binding function as a therapy goal in liver failure: development of a novel adsorbent for albumin dialysis. Ther Apher Dial. 2018 Dec;22(2):196-204.

25 Doumas BT, Watson WA, Biggs HG. Albumin standards and the measurement of serum albumin with bromcresol green. Clin Chim Acta. 1971 Jan;31(1):87-96.

26 Mashige F, Tanaka N, Maki A, Kamei S, Yamanaka M. Direct spectrophotometry of total bile acids in serum. Clin. Chem. 1981 Aug; 27(8):1352-6.

27 Turley SD, Dietschy JM. Re-evaluation of the 3 alpha-hydroxysteroid dehydrogenase assay for total bile acids in bile. J Lipid Res. 1978 Sep;19(7):924-8.

28 Mori L. Modified Jendrassik--Grof method for bilirubins adapted to the Abbott Bichromatic Analyzer. Clin Chem. 1978 Oct;24(10):1841-5.

29 Klammt S, Mitzner SR, Stange J, Loock J, Heemann U, Emmrich J, et al. Improvement of impaired albumin binding capacity in acuteon-chronic liver failure by albumin dialysis. Liver Transpl. 2008 Sep;14(9):1333-9.

30 Büttner S, Patyna S, Koch B, Finkelmeier F, Geiger H, Sarrazin C, et al. Application of hemoadsorption in a case of liver cirrhosis and alcoholrelated steatohepatitis with preexisting hepatitis C infection. Blood Purif. 2017;44(1):30-1.

31 Agar JWM. Review: understanding sorbent dialysis systems. Nephrology. 2010 Jun;15(4): 406-11.

32 Hartmann J, Harm S. Removal of bile acids by extracorporeal therapies: an in vitro study. Int J Artif Organs. 2018 Jan;41(1):52-7.

33 Luo L, Aubrecht J, Li D, Warner RL, Johnson KJ, Kenny J, et al. Assessment of serum bile acid profiles as biomarkers of liver injury and liver disease in humans. PLoS One. 2018 Mar; 13(3):e0193824.
34 Zollner G, Trauner M. Molecular mechanisms of cholestasis. Wien Med Wochenschr. 2006 Jul;156(13-14):380-5.

35 Mouzaki M, Wang AY, Bandsma R, Comelli EM, Arendt BM, Zhang L, et al. Bile acids and dysbiosis in non-alcoholic fatty liver disease. PLoS One. 2016;11(5):e0151829.

36 Peters T. All about albumin biochemistry, genetics, and medical applications. San Diego: Academic Press; 1996.

37 Lee KCL, Baker LA, Stanzani G, Alibhai H, Chang YM, Palacios CJ, et al. Extracorporeal liver assist device to exchange albumin and remove endotoxin in acute liver failure: results of a pivotal pre-clinical study. J Hepatol. 2015 Sep;63(3):634-42.

38 Dominik A, Stange J, Pfensig C, Borufka L, Weiss-Reining H, Eggert M. Reduction of elevated cytokine levels in acute/acute-onchronic liver failure using super-large pore albumin dialysis treatment: an in vitro study. Ther Apher Dial. 2014 Aug; 18(4):347-52.

39 Li M, Cai S-Y, Boyer JL. Mechanisms of bile acid mediated inflammation in the liver. Mol Aspects Med. 2017 Aug;56:45-53.

40 Trivedi PJ, Hirschfield GM, Gershwin ME. Obeticholic acid for the treatment of primary biliary cirrhosis. Expert Rev Clin Pharmacol. 2016 Jan;9(1):13-26.

41 Suraweera D, Rahal H, Jimenez M, Viramontes M, Choi G, Saab S. Treatment of primary biliary cholangitis ursodeoxycholic acid nonresponders: a systematic review. Liver Int. 2017 Dec;37(12):1877-86.

42 Neuschwander-Tetri BA, Loomba R, Sanyal AJ, Lavine JE, Van Natta ML, Abdelmalek MF, et al. Farnesoid X nuclear receptor ligand obeticholic acid for non-cirrhotic, non-alcoholic steatohepatitis (FLINT): a multicentre, randomised, placebo-controlled trial. The Lancet. 2015 Mar;385(9972):956-65.

43 Parés A, Herrera M, Avilés J, Sanz M, Mas A Treatment of resistant pruritus from cholestasis with albumin dialysis: combined analysis of patients from three centers. J Hepatol. 2010 Aug;53(2):307-12.

44 Rozga J, Umehara Y, Trofimenko A, Sadahiro T, Demetriou AA. A novel plasma filtration therapy for hepatic failure: preclinical studies. Ther Apher Dial. 2006 Apr;10(2):138-44.

45 Porowski D, Wirkowska A, Hryniewiecka E, Wyzgał J, Pacholczyk M, Pączek L. Liver failure impairs the intrahepatic elimination of interleukin-6, tumor necrosis factor-alpha, hepatocyte growth factor, and transforming growth factor-beta. BioMed Res Int. 2015; 2015:934065.

46 Pfensig C, Dominik A, Borufka L, Hinz M, Stange J, Eggert M. A new application for albumin dialysis in extracorporeal organ support: characterization of a putative interaction between human albumin and proinflammatory cytokines IL-6 and TNF $\alpha$. Artif Organs. 2016 Apr;40(4):397-402. 